\title{
VIRTUAL REALITY: ITS USEFULNESS FOR ERGONOMIC ANALYSIS
}

\author{
Lawrence E. Whitman \\ Michael Jorgensen \\ Kuresh Hathiyari \\ Don Malzahn \\ Department of Industrial Engineering \& Manufacturing \\ 120 Engineering Building \\ Wichita State University \\ Wichita, KS 67260, U.S.A.
}

\begin{abstract}
This paper presents the results of an effort to compare results of an experiment performed in both a virtual and a real environment. The research question addressed is if virtual reality is a suitable tool for performing ergonomic analysis. The subjects performed a palletizing task in the virtual environment and then performed the same task in the real environment. The results showed that VR can be compared to a similar experimental task in the real environment if it involves measuring only range of movements and no velocities or accelerations. This paper presents these results using a lumbar motion monitor and proposes areas for future improvement and research.
\end{abstract}

\section{INTRODUCTION}

Virtual reality has become a state of art technology for serving a company's future needs in the fields of design, training and organizational communication. It represents reality and allows users to fly, swim, run, and walk through different structures to create usual images in their minds. The promise of virtual reality has such an enormous potential for businesses, from education to government to entertainment, to virtually every form of business imaginable (Patel and Cardinali 1994). Simulations in virtual reality will be able to provide users with a pool of data when used appropriately and at its fullest potential. An advantage of virtual reality is that users do not have to be totally involved to complete the tasks.

The main objective of this paper is to establish an analogy between physical tasks performed in the real world and the virtual world. The basis for this comparison was a manual material handling task that was carried out both in the real environment and the virtual environment. Comparisons of the two environments was accomplished by comparing the torso motion for a palletizing task in both environments.
This paper will describe Virtual Reality, the equipment used, the method, and the results. Finally, some modifications are proposed to make virtual reality more suited to these types of analyses.

\section{VIRTUAL REALITY}

Much history pertaining to virtual reality can be traced back to the early 1950s. There was a development of the "Super Cockpit Project" directed by research teams and this prompted researchers to develop sophisticated devices such as the head mounted display capable of providing the air crew with a computer based stereoscopic image (Stone 1995). The head and eye coordination along with certain brain waves linked to mental task performance was measured (Stone 1995). Further research in the US space program involved the use of Head Mounted Displays (HMD's) and cyber-gloves to monitor the space station maintenance robots and to prepare astronauts to freely walk in space. Because of this and other early developments, VR has gained a foothold in performing experiments all over the world.

Developments in VR were then obtained systematically in the following years. In 1990, British research teams presented their work involving VR at London Computer Graphics Conference (Stone 1995). Virtual reality games became very prominent. But certain researchers believe that applications of virtual reality technologies to children's conceptual learning may not be very advisable (Roussos, et al. 1999). There is a consensus among researchers that VR can have a strong motivational influence. Continuous work involving characterizing phenomena such as immersion and presence are beginning to clarify these effects. VR can extend its opportunities to generate a feel in the environments which for factors of time, distance, scale, and safety, would not be easily accessible to young children especially those that are handi- 
capped (Roussos, et al. 1999). Therefore it is very important that early exposure to virtual environments especially to children be made so that learning capacity and efficiency may well be enhanced.

\subsection{Different Forms of Virtual Reality}

There are three forms of VR namely desktop, projection, and immersion (Schwienhorst 2002). Desktop VR allows the operator to communicate and navigate with the virtual worlds using a wide range of computing equipment. "A typical example of projection VR is the one which presents large screen stereoscopic graphics to groups of individuals by polarizing the images from a pair of conventional video projectors" (Stone 1995). In immersion, the user perceives himself to be totally present in the VR environment by the use of a head mounted display and cyber-gloves. The main aim of immersion is to make use of human skills such as the head and the limb movement, human gestures, stereoscopic vision, hearing, and speech. Efforts are also made so as to make the users avail and exploit the computer technology at the fullest and not be restricted by it (Stone 1995). Users should be able to interact and communicate with the objects in VR in a manner similar to the way they interact and communicate with the objects in the real world.

\subsection{Skill Development in VR}

One of the most significant areas where training has to be devoted is the area of skill development especially in immersive and non-immersive environments. "Skill is defined as the learned ability of associating an optimal action with the task process state or its characteristics" (Adams and Ntuen 1998 1). Skill learning requires acquiring new information time so as to enable and improve a specified job. There are three phases of skill learning. The cognitive phase is where a novice concentrates on understanding the task assigned, the associative phase is where the response patterns emerge while the errors are eliminated, and the autonomous phase is where task completion requires early control (Adams and Ntuen 1998).

Spatial knowledge, displays, and spatial cognition research involve computer simulated three-dimensional environments. People can acquire spatial knowledge by traveling through different environments using maps, photographs, verbal descriptions, in a virtual environment. Three dimensional environments are projected into twodimensional screens of normal desktop computers in desktop systems. In the case of immersive display systems, the output devices such as the HMD increase the impression of being immersed in the virtual environment by preventing the perception of external stimuli from the real environment (Osmann and Berendt 2002). Immersion can affect stress levels within users in the experimental scenario.

Another typical learning application is the estimation of distance. The ability of the user to judge distance accu- rately is essential to many real world tasks which includes navigation, aiming, and shooting. As per previous experiments conducted in direct comparisons of verbal distance, it has been determined that users are less accurate in estimating the distance in VE's than in the real world (Witmer and Sadowski 1998). According to Witmer and Kline (1997), real world estimates average about $75 \%$ of the true distance in the range of 3 to $33 \mathrm{~m}$ whereas in the virtual environment, estimates average about $50 \%$. According to Wright (1995), underestimates in VE ranged from $41 \%$ to $72 \%$ of the true distances, whereas the real world estimates averaged $87 \%$ to $91 \%$ of the true distances (Witmer and Sadowski 1998). Research carried out on this application shows that distance judgments will be accurate when participants are first asked to view an object and then walk to it without further visual guidance. This procedure also known as the non-visually guided locomotion procedure yields errors that are $2 \%$ to $8 \%$ of the true distances at viewing distances up to $22 \mathrm{~m}$ (Witmer and Sadowski 1998). Reiser, et al. (1990) stated that "previously seen targets indicate efferent or proprioceptive information about locomotion and this is closely calibrated to visually perceived distance" (Witmer and Sadowski 1998). Non-visually guided locomotion (NVGL) provides an accurate, unbiased measure of perceived distance unlike the verbal distance estimates. If the virtual environment deforms the perceived distance, NVGL will accurately reflect those deformations. Even though the real world distance judgments obtained via NVGL are more accurate than verbal estimates of distance, extensive research will have to be carried out in order to show that NVGL can produce improved performance on VE's (Witmer and Sadowski 1998).

Given the usefulness of VR and the possibility of skill development using VR, the method of the experiment is now presented.

\section{METHOD}

The main objective was to determine if a correlation exists between virtual tasks and real world tasks for ergonomic analysis purposes. This section describes the experimental design and the procedure involved while carrying out the experiment in the virtual environment and the real environment.

\subsection{Hypothesis Statement}

The primary aspect addressed by this research is whether experiments in the real environment can be performed in the virtual environment and whether data pertaining to the experiment in both environments can be correlated. This research will evaluate the hypothesis that virtual tasks (or experiments) and real world tasks (or experiments) correlate well with each other in terms of data collected and the involvement of the user when performing the experiment. 


\subsection{Equipment}

The equipment used while performing the tasks included: the lumbar motion monitor (LMM), the head mounted display (HMD), and the motion capture system.

\subsubsection{Lumbar Motion Monitor (LMM)}

The lumbar motion monitor is an exoskeleton of the torso that captures the three-dimensional movement of the thoracolumbar spine. The data recorded by the LMM consists of the position, velocity, and acceleration profiles in all the three planes, the sagittal, transverse and the coronal planes, respectively (Marras et al. 1992). The operator with the equipment is shown in figure 1.

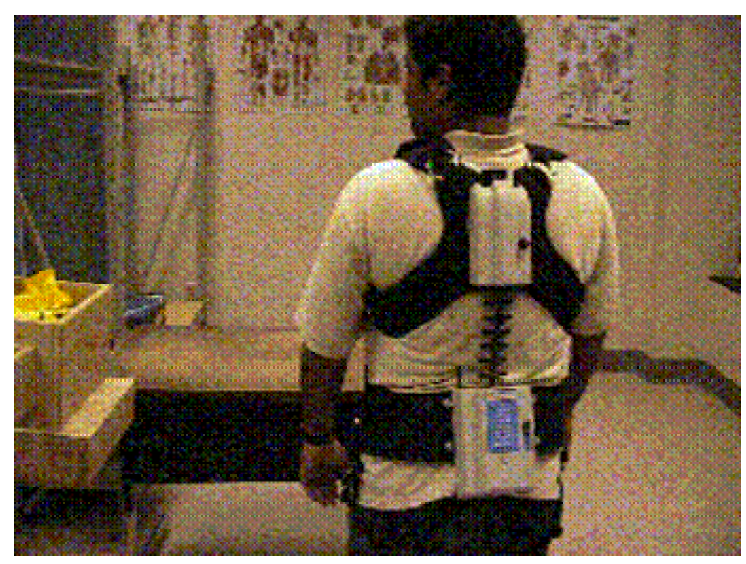

Figure 1: Operator with Lumbar Motion Monitor

\subsubsection{Head Mounted Display (HMD)}

Display devices enable users to navigate through VR very effectively. The HMD is considered a new canonical display. "This device consists of two LCD or CRT screens that are mounted in a helmet-like device so that they are fixed relative to the wearer's eye position" (Bowman, et al. 1999). The HMD views the virtual world by obtaining the user's head position and orientation from a tracking system. An HMD has many attributes which provide the same image to both eyes (biocular) or may provide different images to each eye (stereoscopic). HMD's also come in a wide variety of resolutions which trade-off with the field of view (FOV). The field of view is measured in degrees of horizontal visual angle. A lower FOV causes tunnel vision which may decrease immersion. But in contrast, a higher FOV involves spreading of pixels and this can greatly reduce resolution and introduce distortion. There are also ergonomic issues related to HMD's, such as the display size and the weight as well as the ability to adjust various visual parameters (Bowman, et al. 2000).

Some of the ergonomic challenges associated with the HMD are issues such as the time delay problems. A head coupled system needs to detect, measure, and update visual images according to the head and hand movements, hence a delayed response to the user's movement is predictable. HMD's also have temporary deficits of binocular vision. The causes for this have not been completely explored. Research carried out in this area illustrates that there are a number of factors which interact and make it difficult to determine what causes the deficit in the binocular function (Williams, et al. 1998). Therefore the HMD manufacturers have difficulty knowing how current systems may be modified to prevent any potential visual problems.

\subsubsection{Motion Capture System}

Motion capture can be defined as the recording of movements by an array of video cameras in order to reproduce the exact image in a digital environment. The three dimensional reproduction has many applications in medical assessment of movement disorders, understanding the techniques used in athletics, creating an imaginary person for movies, videogames, broadcast and web cast, and incorporating motion into virtual environments for engineering design. The motion capture system incorporates all the hardware and software applications for complete control and analysis of motion capture (Tebut, Wood, and King 2002). A participant wearing the motion capture suit and performing the experiment is shown in Figure 2.

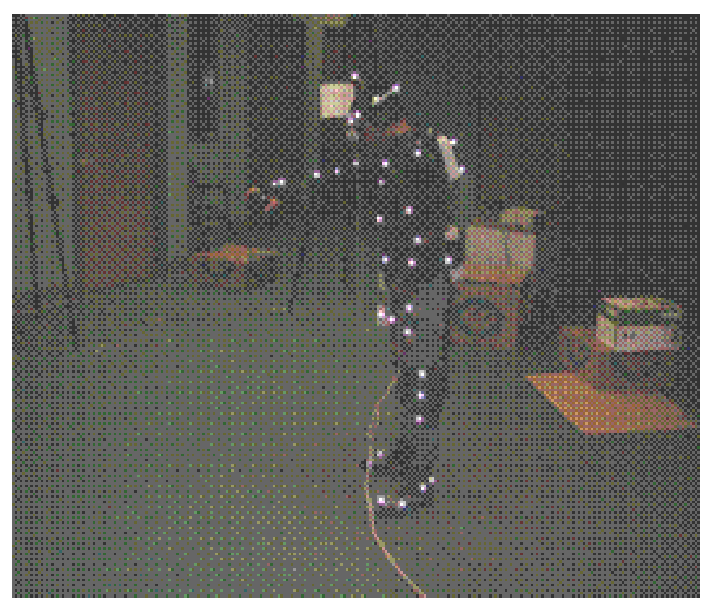

Figure 2: Motion Capture Suit

\subsection{Procedure}

The method included the administration of a presence questionnaire which helped to determine the aspect of presence experienced by the participant. The results of the presence questionnaire are not included in this paper. This paper reports the results of the two sets of experiments which were conducted in each environment. Back motion data was collected via the lumbar motion monitor for both the tasks in both environments. Statistical analysis was 
then used to determine significant differences in data obtained in the virtual and real environments for both the experiments. The two experimental conditions included moving three boxes from one table to another. One experimental condition involved moving three boxes from one table with a height of 15 inches to another table with a height of 15 inches. The second experimental condition was the same except that the origin table height was 38 inches. The destination table remained at 15 inches. Both experimental conditions were performed first in the virtual environment and then in the real environment. Figure 3a shows moving a box between tables with the same height. Figure $3 \mathrm{~b}$ shows moving the boxes between tables with different heights.

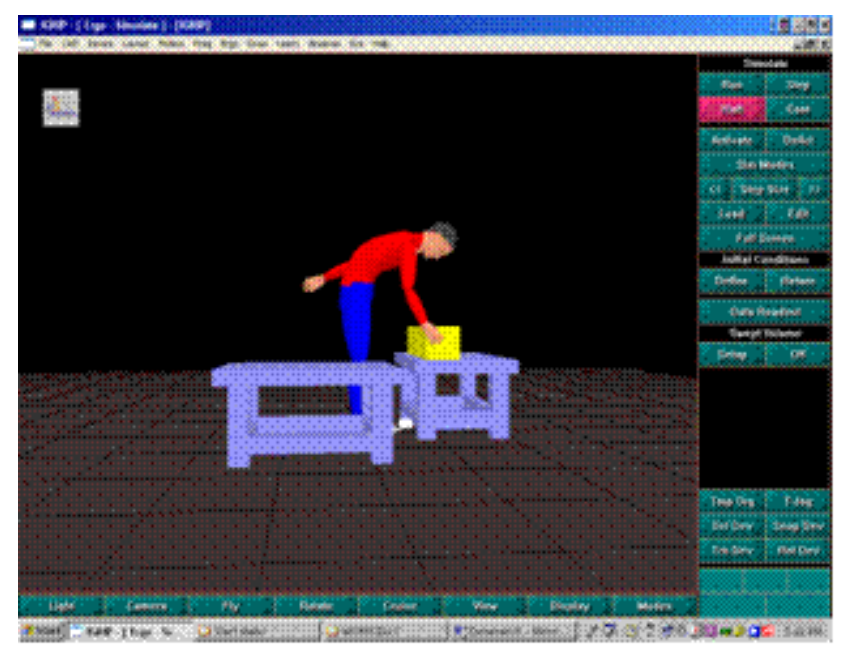

Figure 3(a): Moving a Box between Tables at the Same Height

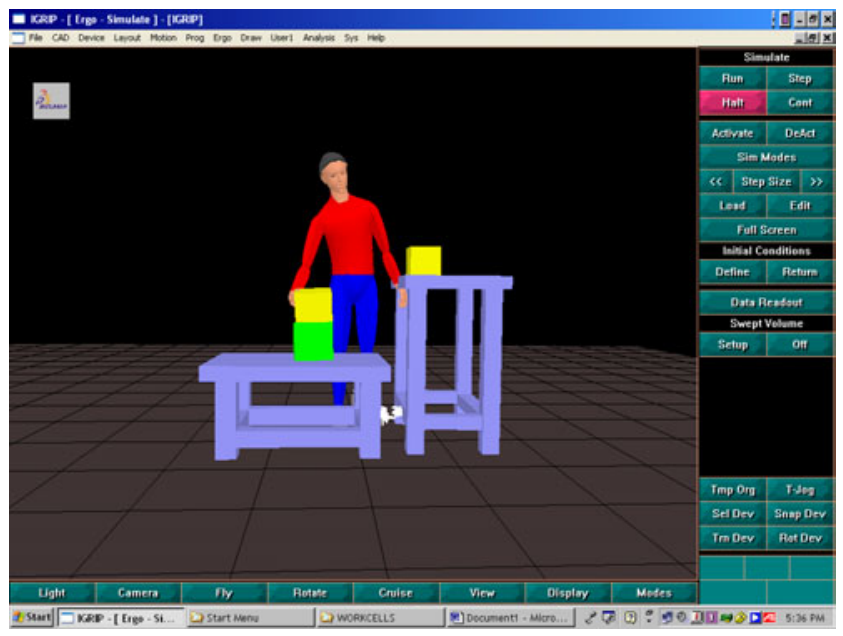

Figure 3(b): Moving a Box between Tables at Different Heights

Figure 4 shows a participant wearing the motion capture suit and the HMD and LMM. Note that there were nine cameras used for capturing motion and the screens are for the observers use only as the participant views the world through the HMD.

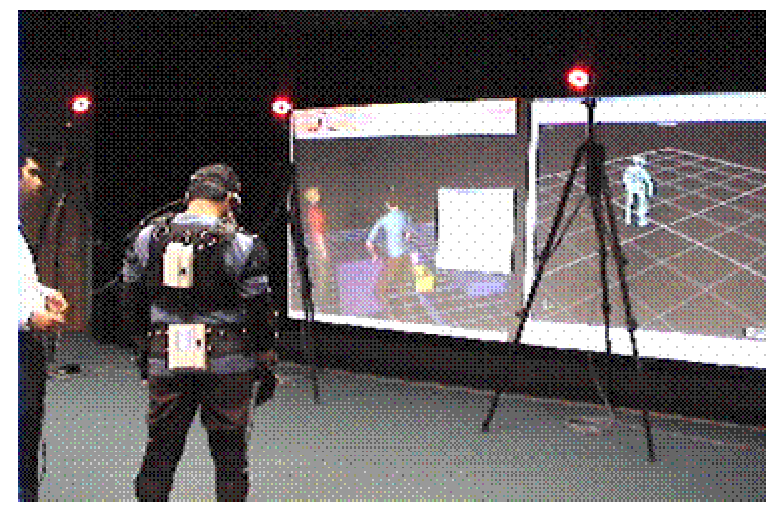

Figure 4: Participant Completely Suited Up

\subsection{Statistical Analysis}

There were nine dependant variables collected from the LMM in each of the two tasks. These are lateral range, sagittal range, twisting angle, maximum lateral velocity, maximum sagittal velocity, maximum twist velocity, maximum lateral acceleration, maximum sagittal acceleration and maximum twist acceleration. There are three boxes. For each box and each dependent variable, a paired t-test analysis was performed to determine if there was a significant difference in data sets between real and VR for that particular independent variable.

\subsection{Results}

From the data gathered, 18 paired t-tests were performed. For Box 1, Box 2, and Box 3 in the first and the second experimental conditions, it was shown that data sets for maximum lateral velocity, maximum sagittal velocity, maximum twist velocity, maximum lateral acceleration, maximum sagittal acceleration and maximum twist acceleration differed significantly between the virtual and the real environments. An example of the complete results for sagittal velocity is shown in Table 1 . For each box the mean values of the virtual environment and the real environment are presented along with the standard deviations. In other words, there was a considerable difference in data sets for velocities and accelerations between virtual and real environments. But for data sets corresponding to maximum lateral range, maximum sagittal range and maximum twisting angle for Box1, Box 2, and Box 3 respectively in the first and the second experiment, it was observed that there was no significant difference in data sets between virtual and real environments. An example of the complete results for sagittal range is shown in Table 2 .

This shows that the hypothesis is rejected for data sets pertaining to velocities and accelerations for the participants while performing experimental tasks in the virtual 
Table 1: Results for Maximum Sagittal Velocity

\begin{tabular}{|c|c|c|c|c|c|c|c|}
\hline \multirow{2}{*}{ Box \# } & \multicolumn{2}{|c|}{ Mean (deg/sec) } & \multicolumn{2}{c|}{$\begin{array}{c}\text { Standard (deg/sec) Deviation } \\
\text { (95\% confidence interval for }\end{array}$} & \multicolumn{2}{c|}{ P value } \\
\hline & VR & Real & VR & Real & Lower limit & Upper limit & \\
\hline Box 1 & 11.97 & 27.35 & 5.14 & 9.66 & -20.953 & -9.794 & $<0.05$ \\
\hline Box 2 & 10.28 & 22.89 & 4.62 & 8.87 & -17.16 & -8.067 & $<0.05$ \\
\hline Box 3 & 10.94 & 25.64 & 5.17 & 8.02 & -19.773 & -9.636 & $<0.05$ \\
\hline
\end{tabular}

Table 2: Results for Maximum Sagittal Range

\begin{tabular}{|c|c|c|c|c|c|c|c|}
\hline \multirow{2}{*}{ Box \# } & \multicolumn{2}{|c|}{ Mean (deg/sec) } & \multicolumn{2}{c|}{$\begin{array}{c}\text { Standard (deg/sec) } \\
\text { Veviation }\end{array}$} & 95\% confidence interval & P value \\
\hline & VR & Real & VR & Real & for difference of means & \\
\hline & & & & & Lower limit & Upper limit & \\
\hline Box 1 & 25.59 & 24.36 & 7.32 & 7.92 & -2.915 & 5.358 & 0.535 \\
\hline Box 2 & 18.29 & 14.14 & 8.71 & 6.17 & -0.354 & 8.652 & 0.068 \\
\hline Box 3 & 16.86 & 16.63 & 7.14 & 6.32 & -3.37 & 3.821 & 0.894 \\
\hline
\end{tabular}

and the real environments. In other words, any experimental task performed in VR is not comparative to a similar experimental task in the real environment which involves velocities and accelerations. This is evidently because the participant moves more slowly in VR as compared to the real environment. There is a lag when observing motion from the HMD in the virtual environment. This causes the motion to be slow moving when viewed by the participant in the HMD. Thus the participant turns, bends, and twists slowly as he/she needs to synchronize his/her pace with the motion viewed. Furthermore, the HMD also has its limitations. The HMD used was monographic and not stereographic. Hence the participant is able to view the motion only in two dimensions and has a difficult time properly perceiving the depth. Again as the participant places the box on the destination table, the participant is not sure of the destination position where he/she is supposed to place the box because of the lag established. Hence the participant's range of movements is slow. Because of this, the participant had to concentrate more on the task being performed and as a result, this caused the participant to move, bend, and twist slowly while performing motion.

Even though we reject the hypothesis for those variables, we fail to reject the hypothesis for data sets pertaining to sagittal, lateral and twisting ranges of the participants while performing experimental tasks in the virtual and the real environments. In other words, any experimental task performed in VR may be comparable to a similar experimental task in the real environment if it involves measuring only range of movements and no velocities or accelerations. In the real environment, the participant knows the exact positions of the origin table, the destination table and the box to be lifted. So the motions are faster.

But in the actual experimental scenario, (industrial or medical applications) a complete comprehensive ergonomic analysis cannot be performed without the velocities and accelerations of movements (ROM) performed by the body.
This involves sagittal, lateral and twist movements in the three dimensional plane.

Hence in the actual experimental scenario we reject the hypothesis and conclude that experimental tasks in the real and the virtual world are not comparable for the devices and equipment used in this research. But this might be made possible with improved technology and more sophisticated devices.

\section{RECOMMENATIONS}

There are several suggestions as to how VR experiments can be made more realistic:

1. Software interface - improved processing speeds would help in measuring velocities and accelerations in VR.

2. Feedback mechanism - a haptic or force feedback mechanism would enable the touch sensation and would enable the participant to experience a more realistic environment.

3. Stereo HMD - a stereo HMD should be used to perceive the object depth. A stereo HMD will have high accuracy, fine resolution, and high frequency. The improved HMD will also lower the latency and it will be able to work over wider areas.

Clearly, the main difficulty in achieving a virtual environment that closely simulates the real environment is the time lags. Once these can be minimized sufficiently (and depth perception issues are properly address), virtual environments can be used for ergonomic analysis purposes.

\section{ACKNOWLEDGMENTS}

This material is based upon work supported by the National Science Foundation under Grant No. 0125414. Any 
opinions, findings, and conclusions or recommendations expressed in this material are those of the author(s) and do not necessarily reflect the views of the National Science Foundation.

\section{REFERENCES}

Adams, K.A. and C.A. Ntuen. 1998. Skill training in immersive and non immersive environments. Department of Industrial \& Systems Engineering, North Carolina Agricultural and Technical State University, 1-6.

Bowman, D.A., A. Datey, Y.S. Ryu, U. Farooq, and O. Vasnaik. 1999. Empirical comparison of human behavior and performance with different display devices for virtual environments. Grado Department of Industrial and Systems Engineering, Virginia Polytechnic Institute and State University, 1-5.

Marras, W.S., F.A. Fathallah, R.J. Miller, S.W. Davis, and G.A. Mirka. 1992. Accuracy of a three-dimensional lumbar motion monitor for recording dynamic trunk motion characteristics, International Journal of Industrial Ergonomics, 9, 75-87.

Osmann, P.J. and B. Berendt. 2002. Investigating distance knowledge using virtual environments. Environment and Behavior, 34, 178-193.

Patel, H., and R. Cardinali. 1994.Virtual Reality Technology in Business. Management Decision, 32, 5-12.

Roussos, M., A. Johnson, T. Moher, J. Leigh, C. Vasilakis, and C. Barnes. 1999. Learning and building together in an immersive virtual world. Presence, 8, 247-263.

Schwienhorst, K. 2002. Why virtual, why environments? Implementing virtual reality concepts in Computer Assisted Language Learning. Simulation and Gaming, 33, 196-209.

Stone, R.J. 1995.The reality of virtual reality. World class design to manufacture, 2: 11-17.

Tebutt, P., J. Wood, and M. King. 2002. The Motion Capture Manual. Copyright (C) 2002 OMG Plc.1-15.

Williams, M.M., A. Plooy, R.B. Limerick, and J. Wann. 1998. Gaze angle: A possible mechanism of visual stress in virtual reality headsets. Ergonomics, 41, 280-285.

Witmer, B.G. and W.J. Sadowski. 1998. Nonvisually guided locomotion to a previously viewed target in real and virtual environments. (Special Section: Virtual environments: Models, Methodology, and Empirical Studies). Human Factors, 40, 478-485.

\section{AUTHOR BIOGRAPHIES}

LAWRENCE E. WHITMAN, Ph. D., P. E. is Associate Professor in the department of Industrial and Manufacturing Engineering at Wichita State University. He joined WSU in 1999 after 13 years in the aerospace industry. He has a B.S. in Mechanical Design Engineering Technology from Oklahoma State University (1984), a M.S. in Indus- trial Engineering from Oklahoma State University (1986) and a Ph.D. in Industrial Engineering from the University of Texas at Arlington (1999). His research interests include enterprise modeling, lean manufacturing and supply chain design. He is a member of IIE and APICS. His e-mail address is <larry.whitman@wichita.edu> .

MICHAEL J. JORGENSEN, Ph. D. is an Assistant Professor in the department of Industrial and Manufacturing Engineering at Wichita State University. He received his B.S. (1986) and M.S. (1989) in Industrial Engineering from the University of Nebraska and a Ph.D. (2001) in Industrial and Systems Engineering from The Ohio State University. He has published over twenty journal and conference articles in the areas of industrial ergonomics and occupational biomechanics. His e-mail address is <michael.jorgensen@wichita.edu> .

KURESH HATHIYRAI is a graduate student in the department of Industrial and Manufacturing Engineering at Wichita State University. He received his BS in Production Engineering, Mumbai University, India and is pursuing his M.S in Industrial Engineering in the department of Industrial and Manufacturing Engineering at Wichita State University. He joined WSU in Fall 2001 after working for 2 years in aluminum tube and automobile bearing companies in Mumbai, India. He is a graduate teaching and research assistant and an active member of the APICS where he served as the director of programs. His areas of interests are materials management, supply chain and lean manufacturing.. His email address is <kureshh@hotmail.com> .

DON E. MALZAHN, Ph. D. is a Professor in the department of Industrial and Manufacturing Engineering at Wichita State University. He received his BS, MS, and $\mathrm{Ph} . \mathrm{D}$. in Industrial Engineering from Oklahoma State University. He has over 28 years teaching experience with research interests in engineering education, rehabilitation engineering, decision analysis, and project management. His e-mail address is <don.malzahn@wichita.edu> . 